\title{
Altered Branching of Serotonin-Containing Neurons in Drosophila Mutants Unable to Synthesize Serotonin and Dopamine
}

\author{
Vivian Budnik ${ }^{1, a}$ Chun-Fang $W u^{3}$ and Kalpana White ${ }^{2}$ \\ 'Biophysics Program and 'Department of Biology, Brandeis University, Waltham, Massachusetts 02254, and ${ }^{3}$ Department \\ of Biology, University of lowa, lowa City, lowa 52242
}

\begin{abstract}
The anatomy of peripheral serotonin-containing fibers (5-HT fibers) in the gut of wild-type Drosophila larvae was compared to mutants deficient in the gene that encodes the enzyme dopa decarboxylase (DfDdc mutants). The 5-HT fibers, located in the proventriculus and midgut, were visualized immunocytochemically by using a monoclonal antibody against 5-HT. Since DfDdc larvae are devoid of 5-HT and dopamine in the nervous system, the highly selective uptake capability of 5-HT neurons was used to visualize the 5-HT fibers. We found that the absence of 5-HT and dopamine in the nervous system of DfDdc animals does not prevent 5-HT fibers from reaching their appropriate targets. However, these fibers in the mutant show a 2-fold increase in the extent of branching. This effect is specific to 5-HT fibers, since glutamate-like and FMRFamide-like immunoreactive fibers of the proventriculus and midgut remain unaffected in the mutant. Low but detectable levels of dopamine and 5-HT in the CNS are sufficient to prevent the increase in arborization, as indicated by analyses of a temperaturesensitive $D d c$ allele $\left(D d c^{t s 2}\right)$, which has very low dopa decarboxylase activity. The abnormally extensive branching of 5-HT fibers also can be partially rescued by feeding DfDdc larvae with dopamine. In contrast, feeding with a 5-HT-containing diet had no effect on the mutant phenotype. Hypotheses that could explain the mutant phenotype are proposed.
\end{abstract}

The signals involved in the formation of the highly precise architecture of the nervous system are largely unknown. In recent years, however, it has become increasingly clear that multiple factors contribute to the general assembly of developing neural circuits. Some of the signals that have been shown to have some relevance in neurogenesis are nerve growth factor, cell surface molecules, elements in the extracellular matrix, electrical activity, and electrical fields (reviewed in Harris, 1982). Another set of molecules that has been suggested as regulators of neural development and differentiation are neurotransmitters (Harris, 1982; Lauder and Krebs, 1984). Pharmacological experiments

\footnotetext{
Received Aug. 29, 1988; accepted Jan. 18, 1989.

We wish to thank Drs. C. Toomim and P. Petrusz for a generous gift of glutamate antiserum. The FMRFamide antiserum was kindly provided by Dr. E. Marder. We appreciate helpful comments on the manuscript from Drs. M. Gorczyca and J. Engel. This work was supported by NIH grant NS 23510. Part of this work was submitted by V.B. in partial fulfillment of the requirements for the degree of Ph.D. at Brandeis University.

Correspondence should be addressed to Vivian Budnik, Department of Biology, University of Iowa, Iowa City, IA 52242.

${ }^{a}$ Present address: Department of Biology, University of Iowa, Iowa City, IA 52242.

Copyright (c) 1989 Society for Neuroscience $0270-6474 / 89 / 082866-12 \$ 02.00 / 0$
}

both in vitro and in vivo lend support to this hypothesis. For example, in rats, 5-HT depletion retards the onset of neuronal differentiation in fetal brain regions that, in the adult, receive serotonergic innervation (Lauder and Krebs, 1978). In the cat visual cortex, the experiments of Pettigrew and Kasamatsu (1976) suggested that alterations in the levels of norepinephrine affect the plasticity in this region. Later, it was found that this effect was associated with lesions of the cholinergic system (Bear and Singer, 1986).

The work of Kater et al. demonstrates that the growth and connectivity of specific identified neurons of the buccal ganglion of Helisoma can be affected by 5-HT and dopamine. Addition of 5-HT to the culture medium causes an immediate cessation of growth cone movements and neurite elongation of specific neurons. In addition, this neurotransmitter is able to affect synaptogenesis by preventing certain identified neurons from forming electrical synapses with other neurons (Haydon et al., 1984, 1987; Mercier et al., 1985). These in vitro studies are supported by pharmacological studies in vivo. Pharmacological agents that lead to transient reduction in 5-HT content during a defined period in embryogenesis have been shown to cause an increase in the number of branches in an identified neuron whose growth was inhibited by addition of 5-HT to the culture medium (Goldberg and Kater, 1989). Similarly, dopamine can act to suppress regenerative outgrowth of another population of specific neurons (McCobb et al., 1988).

The fruit fly Drosophila melanogaster provides a unique in vivo system in which to study the role of neurotransmitters in the development of the nervous system. By generating mutants with defined lesions, it is possible to manipulate neurotransmitter levels during different periods, or continuously throughout development (Greenspan, 1980; Greenspan et al., 1980; Budnik et al., 1986; Vallés and White, 1986; Budnik and White, 1987a; Gorczyca and Hall, 1987). We have been studying the biogenic amine system in Drosophila (Budnik and White, 1988; Vallés and White, 1988), since by using mutations that eliminate the gene that codes for the enzyme dopa decarboxylase (DDC), it is possible to generate animals devoid of 5-HT and dopamine in the CNS ( $D f D d c$ mutants; reviewed in Hirsh, 1986; Wright, 1987). In addition, by using mutations in the gene pale (ple), which presumably codes for the enzyme tyrosine hydroxylase, mutants with no dopamine but with unaltered 5-HT synthesis can be generated (Budnik and White, 1987a).

Vallés and White (1986) described the pattern of 5-HT-containing neurons (5-HT neurons) in Drosophila larval CNS. This pattern also was investigated in $D f D d c$ animals by using $5-\mathrm{HT}$ uptake. They found that although the mutant CNSs were devoid of 5-HT, 5-HT neurons differentiated and retained their capac- 
ity to uptake 5-HT. The main conclusion of those studies was that the absence of 5-HT throughout development did not alter the pattern of distribution of neurons normally committed to express 5-HT. This observation raises several questions regarding the differentiation of 5-HT neurons in mutant CNSs. Is differentiation of 5-HT neurons in the mutant identical to that in the wild type? Do they contact with their normal targets? Are their dendritic arbors unchanged?

In this paper, we examine these questions by looking at 5-HT-containing fibers and nerve terminals in Drosophila larval gut. 5-HT fibers in this region are present at low density without profuse overlap, making this preparation amenable to analysis. Our major finding is that $D f D d c$ animals show substantially increased arborization of 5-HT nerve terminals in the gut. Although the exact cellular and molecular mechanisms responsible for excessive arborization are unknown, this finding lends further support to the hypothesis that neurotransmitters might regulate neuronal connectivity in addition to their classic role in synaptic transmission. Some of these studies have been presented in abstract form (Budnik et al., 1988).

\section{Materials and Methods}

Fly cultures and strains. Unless otherwise specified, all flies were raised at $25^{\circ} \mathrm{C}$ on standard medium. The strain Oregon- $R(O R)$ was used as wild-type control. For comparative purposes, the wild-type strain Canton-S $(C S)$ also was used. The mutant chromosomes used are listed below; the mutations that served as markers on these chromosomes have been described previously (Lindsley and Grell, 1968).

Df(2L)TW 130, rdo pr cn: A cytologically visible deletion that completely removes the gene $D d c$ (Wright et al., 1976) marked with $r d o, p r$, and cn

$D d c^{n 27} p r$ : A 2-kb deletion removing the two $5^{\prime}$ exons of $D d c$ and 0.5 $\mathrm{kb}$ of $5^{\prime}$ flanking DNA (Gilbert et al., 1984), marked with $p r$

$D d c^{s 2}$ : Ethylmethane sulphate (EMS)-induced temperature-sensitive allele (Wright et al., 1981)

$\mathrm{CyO}$ : A second chromosome balancer marked with $\mathrm{dpl} \mathrm{Cy} \mathrm{pr} \mathrm{cn}^{2}$

Studies in animals deficient in the gene Ddc. To generate $D f D d c \mathrm{mu}$ tants, the genetic cross $D f(2 \mathrm{~L}) 130 / C y O \times D d c^{n 27} / C y O$ was used. $D f D d c$ mutants normally die at the embryo-larval boundary. Nevertheless, they can survive up to a late larval stage if they are mechanically hatched. These mutant animals can be recognized by their unpigmented mouth parts and setae. Mechanically hatched animals were reared in enriched larval medium (see Vallés and White, 1986). For 5-HT, dopamine, or $\mathrm{N}$-acetyldopamine feeding experiments, $200 \mu \mathrm{l}$ of freshly made amine was added to enriched larval medium to give a final concentration of $10^{2} \mathrm{M} . D / D d c$ larvae were transferred every day to new vials containing enriched larval medium into which freshly made amine solution, or water alone, was mixed.

Studies of temperature-sensitive Ddc allele ( $\mathrm{Ddc}^{\mathrm{ts} 2}$ ). To decrease DDC enzyme activity in $D d c^{s s}$ mutants, broods were shifted to $30^{\circ} \mathrm{C}$ starting at least $1 \mathrm{~d}$ before egg collections. Eggs were collected and staged as described in Vallés and White (1986) and allowed to develop until hatching. Newly hatched larvae were transferred to enriched larval medium. The incubation temperature was maintained at $30^{\circ} \mathrm{C}$ throughout development until dissection at the third instar stage.

Immunocytochemistry. The method used was the indirect immunocytochemical technique using a secondary antibody coupled to fluorescein isothiocyanate (FITC; Cappel) at a 1:20 dilution. As a primary antibody, a 5-HT monoclonal antibody (Accurate Chemical and Scientific Corp., Clone YC5/45) at a 1:100 dilution was used. For detailed immunocytochemical protocol and controls for antibody specificity, see Vallés and White (1986). Samples were stored at $-70^{\circ} \mathrm{C}$. Under these conditions, the fluorescence remained robust for many months.

For analyzing FMRFamide-like immunoreactivity (White et al., 1986), a polyclonal antibody against the peptide (antibody $671 \mathrm{C}$ of Marder et al., 1987) at a 1:600 dilution was used. The secondary antibody was goat antirabbit IgG coupled to FITC at a 1:20 dilution.

For glutamate immunocytochemistry, the protocol of Johansen et al. (1989) was adopted with the following modifications: (1) dissections were done in Ikeda's saline (110 mM NaCl, $4.7 \mathrm{~mm} \mathrm{KCl}, 1.8 \mathrm{mM} \mathrm{CaCl}_{2}$,

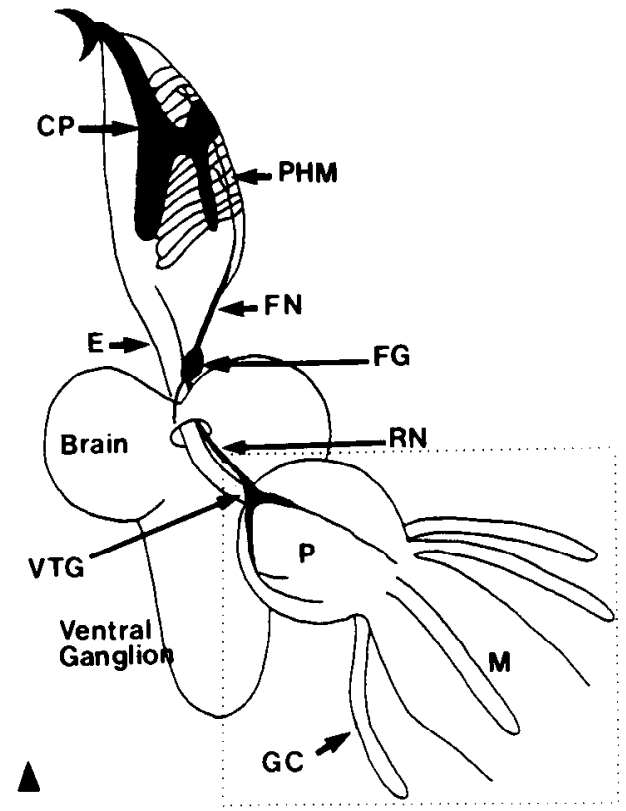

Figure 1. Schematic representation of the anterior region of the digestive system of Drosophila larvae. The tracing is an adaptation from the whole-mount preparation shown in Figure 2. The region enclosed by stippled lines corresponds to the proventricular-midgut region shown in Figure $6, A, B$. Arrowhead at bottom left points to anterior. $C P$, cephalopharyngeal plates; $E$, esophagus; $F G$, frontal ganglion; $F N$, frontal nerve; $G C$, gastric caeca; $M$, midgut; $P$, proventriculus; $P H M$, pharyngeal muscles; $R N$, recurrens nerve; $V T G$, ventricular ganglion.

$0.74 \mathrm{mM} \mathrm{KH}_{2} \mathrm{PO}_{4}, 0.35 \mathrm{mM} \mathrm{Na}_{2} \mathrm{IPO}_{4}, \mathrm{pH} 7.0$ ), and (2) washes were done in $0.1 \mathrm{M}$ phosphate buffer, $\mathrm{pH} 7.2,0.3 \%$ Triton X-100. It was found that incubation with goat nonimmune serum made no significant difference in the immunoreactivity and background of the preparations. Therefore, in most experiments, nonimmune serum was not used. The specificity of the primary antibody is demonstrated by Hepler et al. (1988) and by Johansen et al. (1989). The dilution of the primary antibody was 1:400. Secondary antibodies were goat antirabbit immunoglobulin conjugated either to FITC or tetramethyl rhodamine isothiocyanate (TRIC).

For uptake experiments, dissected CNSs and digestive tissues were incubated for $1 \mathrm{hr}$ at room temperature in Ikeda's saline containing $10^{-6}$ M 5-HT (Sigma). Then the tissues were rinsed 3 times with cold, calcium-free saline and processed for 5 -HT immunocytochemistry as described.

Quantification of varicosities and branches in the midgut. The boundary between the proventriculus and midgut can be identified easily as an indentation between these organs. Branch points and varicosities were counted in the region posterior to that indentation (see Fig. 6, arrows). A varicosity was defined as a discrete enlarged region of the neurite. Varicosities were unambiguously identifiable because of their brighter fluorescence (see Results and Fig. 6C). Counts of branch points and varicosities were expressed as the mean \pm SEM. The means were compared using the Student's $t$-test.

Cuticle preparation. To visualize the pigmentation of cuticular structures in the larva, the larval cuticle was processed according to Van der Meer (1977). Briefly, larvae were pricked in glycerol: acetic acid (1:4) and incubated in this solution overnight at $60^{\circ} \mathrm{C}$. The samples were then mounted in Hoyer's solution and incubated at $45^{\circ} \mathrm{C}$ for $24 \mathrm{hr}$ beforc observation.

\section{Results}

The foregut of Drosophila larvae consists of the pharynx, the esophagus, and the proventriculus (Fig. 1). The proventriculus is a spherically shaped organ associated with trituration. The stomodeal valve, which controls entry of food to the midgut, is located at the posterior end of the proventriculus. An enlarged 


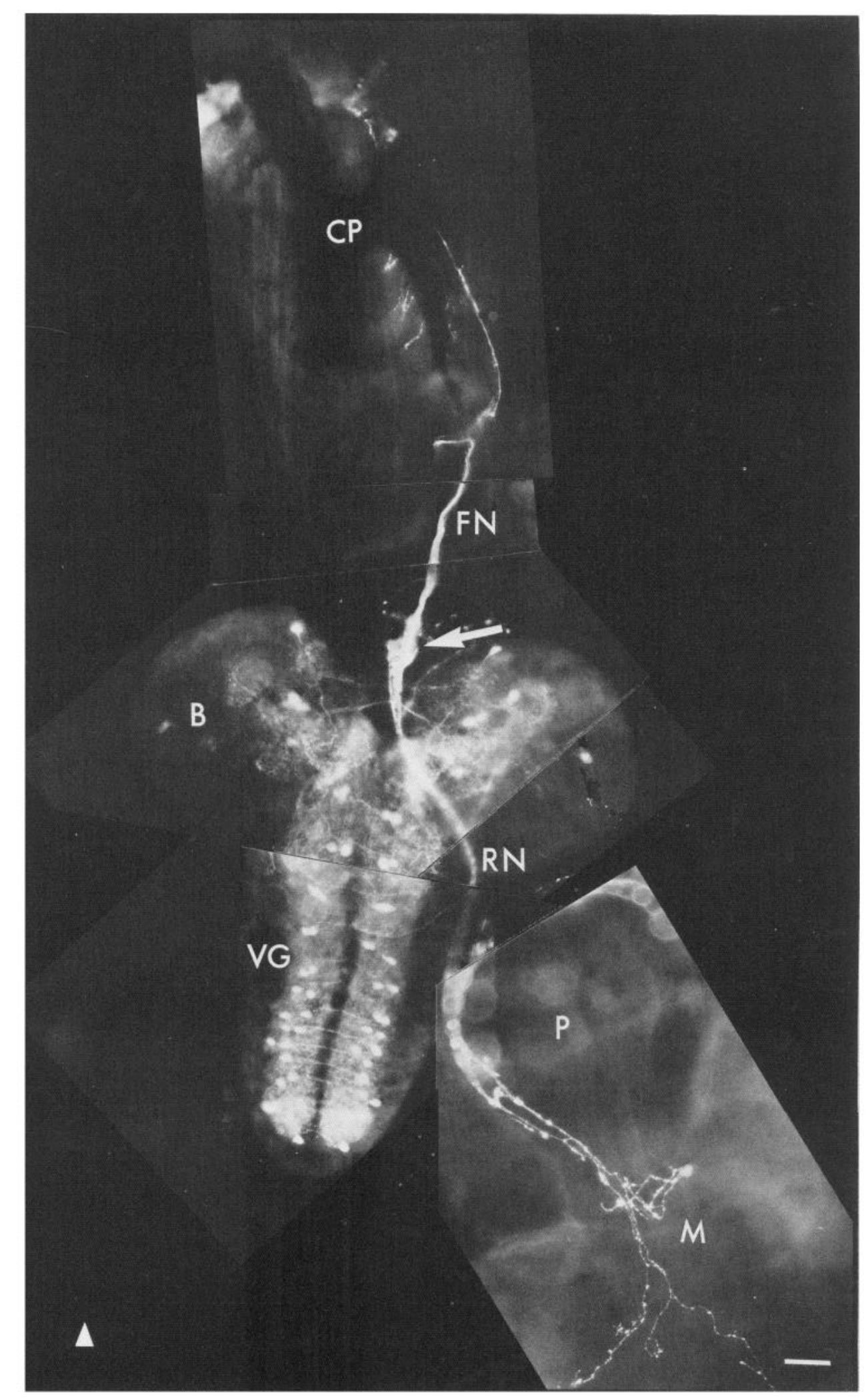

Figure 2. Distribution of 5-HT immunoreactivity in the CNS and digestive system of a third instar wild-type larva. Notice the high levels of immunofluorescence in the frontal nerve $(F N)$, frontal ganglion (arrow), and recurrens nerve $(R N)$. Also notice fluorescent fibers in the proventriculus $(P)$ and midgut $(M)$. For a description of 5 -HT immunoreactive neurons in the brain $(B)$ and ventral ganglion $(V G)$, see Vallés and White (1988). CP, cephalopharyngeal plates. Scale bar, $40 \mu \mathrm{m}$. Arrowhead at lower left points to anterior.

portion of the midgut follows the proventriculus. Between the proventriculus and midgut, there are 4 finger-like evaginations, termed the gastric caeca, that are loci for most digestive enzymes (Bodenstein, 1950; Campos-Ortega and Hartenstein, 1985). The foregut and midgut are innervated by 3 peripheral ganglia, which compose the stomatogastric nervous system: the frontal ganglion, which lies posterior to the cephalopharyngeal apparatus, the hypocerebral ganglion, behind the transverse commissure of the brain, and the ventricular ganglion, located in the anterior portion of the proventriculus. The frontal ganglion connects to 

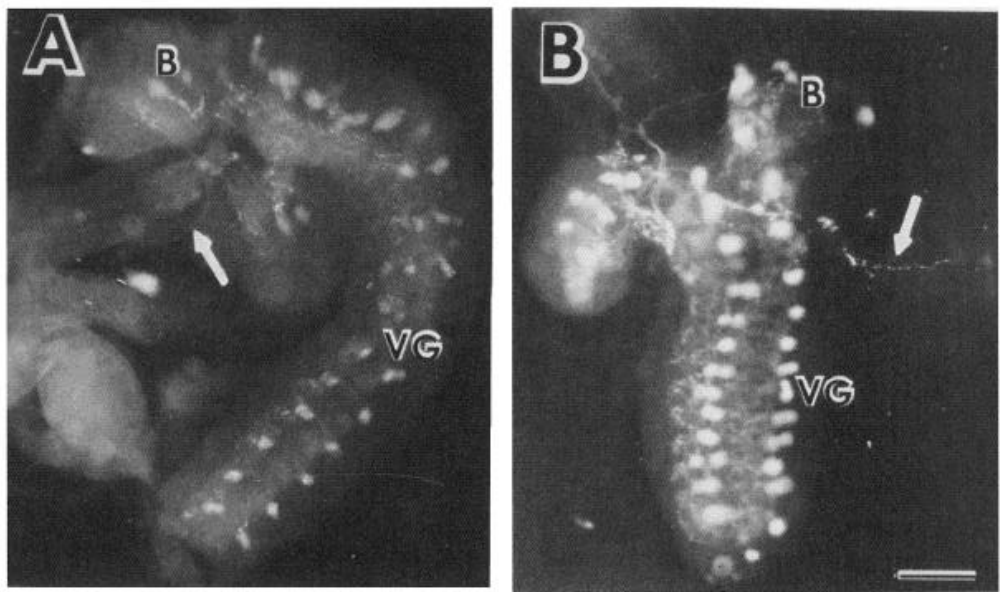

Figure 3. Development of 5-HT-containing fibers in the proventriculus and midgut. $A$, Immunoreactivity in 5 - $\mathrm{HT}$ neurons in the CNS of a $16-18-\mathrm{hr}$ embryo. Note the dimly fluorescent cell bodies and the absence of immunoreactivity in the proventriculus (arrow). $B$, Immunoreactivity in the nervous system of a $20-22-h r$ embryo. Notice the immunoreactivity in the proventriculus (arrow). $B$, brain; $V G$, ventral ganglion. Scale bar, $40 \mu \mathrm{m}$.

the brain and to the pharyngeal muscles by the unpaired frontal nerve and to the ventricular ganglion, proventriculus, and midgut by the recurrens nerve (Campos-Ortega and Hartenstein, 1985; this report).

In the following sections, we describe the distribution and development of 5-HT immunoreactive fibers and cells in the proventriculus and midgut region. In addition, we describe immunoreactivity for 2 other neuroactive molecules: glutamate and FMRFamide-like peptides. These immunoreactivities are then examined in $D f D d c$ mutants.

5-HT. 5-HT-immunoreactive fibers are present in both the proventriculus and the anterior midgut (Fig. 2). These fibers most likely originate from 5-HT neurons in the brain and/or frontal ganglion, since 5-HT-containing cell bodies sending processes to the recurrens nerve are observed at these sites. Profuse 5 -HT-containing fibers and varicosities are present in both the frontal and recurrens nerve (Fig. 2; Budnik and White, 1987b). The 5-HT-containing fibers in the recurrens nerve enter the anterior end of the proventriculus, near its junction with the esophagus. These fibers are discerned as single fibers or 2-4 fasciculating fibers that run along the anterior-posterior axis of the proventriculus and enter the midgut. At the level of the anterior midgut, these fibers ramify (Fig. 2). No 5-HT fibers are observed in the gastric caeca. A characteristic feature of the 5-HT fibers is the large varicosities present throughout the entire length, giving the fiber a beaded appearance (Figs. 2, 6C).

Development of 5-HT fibers. Embryogenesis in Drosophila lasts about $22 \mathrm{hr}$ at $25^{\circ} \mathrm{C}$. 5-HT-immunoreactive cell bodies and projections appear by late embryogenesis when the basic organization of the CNS is complete. The pattern of distribution of 5-HT neurons consist of a metameric, bilaterally symmetrical array of neurons in the ventral ganglion that has been described by Vallés and White (1986; also see Figs. 2, 3). The pattern of 5 -HT fibers in the proventriculus and midgut is at least partly complete before hatching.

By 16-18 hr of embryogenesis, 5-HT-immunoreactive cell bodies and primary projections are observed in the CNS. However, very few neuropil ramifications and no peripheral 5-HT fibers are present at this time (Fig. 3A). By 18-20 hr 5-HTimmunoreactive fibers and varicosities are seen in the CNS and, in few samples, in the recurrens nerve. Since at this stage tissues are extremely small and fragile, failure to observe 5-HT im- munoreactivity in the recurrens nerve could be due to damage of the tissue during manipulation. The proventriculus and midgut were completely devoid of immunoreactivity in all $11 \mathrm{sam}$ ples observed. By 20-22 hr, 5-HT-immunoreactive fibers were distributed along the proventriculus and midgut in most samples observed (8/10; Fig. $3 B$ ). Since the size of the proventriculus and midgut increases considerably during the larval period (e.g., the diameter of the proventriculus in a pharate larva is about $30 \mu \mathrm{m}$, whereas in a third instar larva, it is about $200 \mu \mathrm{m}), 5-$ HT fibers must continue extending during the larval stages. This is evident when the projections of a 22-hr embryo and a third instar larva are compared (compare Fig. $3 B$ with Fig. 2). The extent of this growth, as well as its developmental time course, remains to be further investigated.

Glutamate. Glutamate-like immunoreactivity is observed in fibers of the proventriculus, midgut, and gastric caeca and in a regular array of cells in the gastric caeca. Unlike 5-HT, which shows a restricted distribution to a few fibers, glutamate-like immunoreactivity is profuse (Fig. 4).

The pattern of distribution of glutamate-containing fibers in the proventriculus is similar to the 5 -HT pattern (compare Fig. 2 with Fig. 4A). However, glutamate fibers do not have the varicosities characteristic of 5-HT fibers at these sites. Glutamate fibers of the proventriculus could originate in the ventricular ganglion, since 2 immunoreactive cell bodies are sometimes observed at this site (not shown). In the midgut, glutamate immunoreactivity is observed in longitudinal fibers and varicosities along muscle bands (Fig. $4 B$ ). This gives to the midgut a regular "striped" appearance. In the gastric caeca, 1 or 2 rows of unpaired immunoreactive cells (GC cells) are observed (Fig. $4 C$ ). In addition, glutamate immunoreactivity is distributed in longitudinal and transverse fibers or bundles. Some of the transverse fibers seem to originate from the GC cells (Fig. 4C).

FMRFamide-like peptide(s). The pattern of neurons containing FMRFamide-like peptides in Drosophila has been described by White et al. (1986). FMRFamide-immunoreactive fibers are observed in the proventriculus, midgut, and gastric caeca (Fig. 5 ). This distribution is very similar to the 5-HT pattern in the proventriculus and midgut. However, they represent distinct sets of fibers, as revealed by double-labeling experiments (not shown). In the gastric caeca, profuse immunoreactive varicosities are observed. In addition, immunoreactivity is observed 

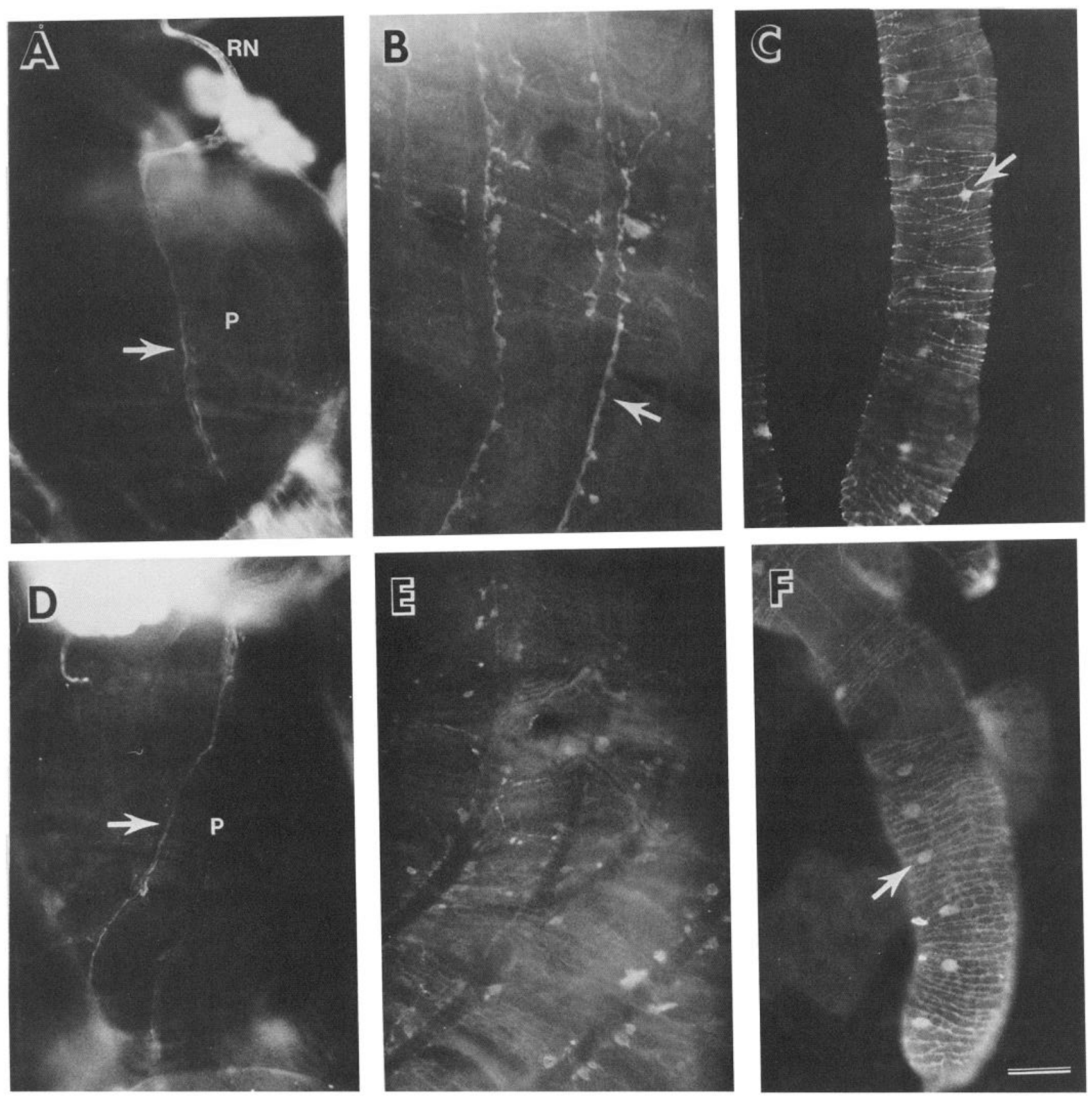

Figure 4. Distribution of glutamate-like immunoreactivity in the gut of $(A, B, C)$ a third instar wild-type larva and $(D, E, F)$ a third instar mutant larva. $A, D$, Glutamate-containing fibers (arrow) in the proventriculus $(P)$. Notice the fluorescence in the recurrens nerve $(R N)$. $B, E, G$, Glamatecontaining fibers and varicosities in the midgut. Arrow points to one of the longitudinal fibers observed at this region. $C$, $F$, Glutamate-like immunoreactivity in the gastric caeca. Note the presence of gastric caecal cells (arrow). Scale bar, $40 \mu \mathrm{m}$ in $A, C, D, F$, and $15 \mu \mathrm{m}$ in $B, E$.

in about 4-6 cells in the ventricular ganglion (Fig. $5 A$; see also White et al., 1986) and in the neuropil of the frontal ganglion (not shown).

\section{DfDdc mutants exhibit abnormal arborization of 5-HT} neurons

In Drosophila, DDC enzyme activity is necessary for the decarboxylation of L-dopa to dopamine and of 5-hydroxytryptophan to 5-HT in the CNS (Livingstone and Tempel, 1983). In addition, this enzyme is necessary for tanning and sclerotization of the larval and adult cuticle. Animals deficient in the gene $D d c$ can develop and live as far as the late larval stages.

$D f D d c$ mutants completely lack 5-HT in the CNS (Vallés and White, 1986; Johnson et al., 1987). However, incubation of mutant CNSs in exogenous 5-HT reveals a pattern of immunoreactivity indistinguishable from wild type. This result indicates that the uptake system of 5-HT neurons in Drosophila is highly cell-specific and can be employed as a way to visualize 

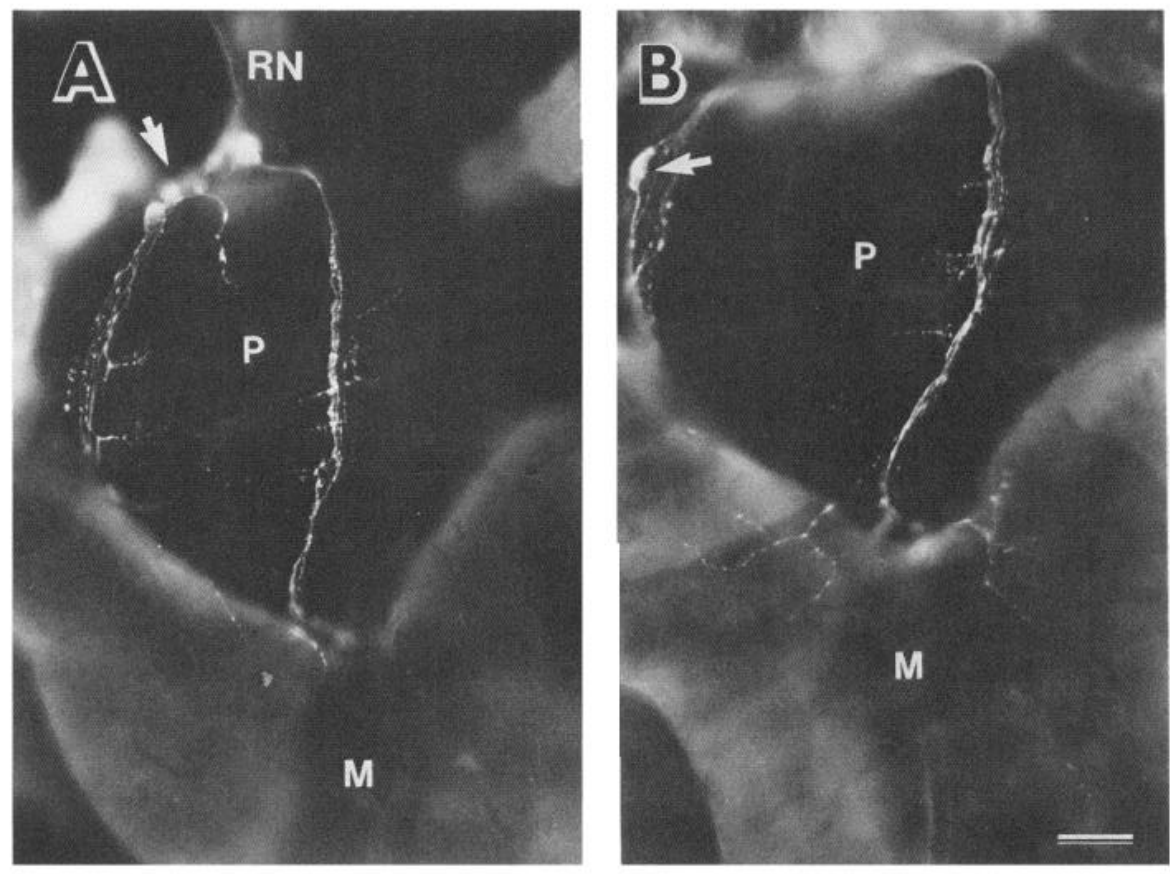

Figure 5. Distribution of FMRFamide-containing fibers in the gut of $(A)$ a third instar wild-type larva and $(B)$ a third instar mutant larva. Arrows point to neurons at the ventricular ganglion. $M$, midgut; $P$, proventriculus; $R N$, recurrens nerve. Scale bar, $40 \mu \mathrm{m}$. and analyze 5-HT neurons in "serotonin-less" mutants. In the following sections, we look at the pattern of branching of 5-HT neurons in the proventriculus and midgut.

In $D f D d c$ mutants, fibers in the proventriculus and midgut are able to take up 5-HT. These fibers must correspond to the 5-HT-containing fibers in the wild type, since the 5-HT uptake system in Drosophila is cell-specific (see above), and the mean number of 5-HT branches and varicosities in the proventriculus and midgut of wild-type preparations is not altered by incubation in exogenous 5-HT. In fact, the mean number of varicosities and branches in wild-type samples incubated in 5-HT was $129 \pm 15$ and $21.5 \pm 2.0(n=11)$, respectively. In wildtype samples without 5 -HT incubation, they were $122 \pm 13$ and $22.1 \pm 1.5(n=10)$.

A striking feature of 5-HT projections in the proventriculus and midgut of $D f D d c$ mutants is an abnormally extensive ramification of fibers. In addition, fibers extend more posteriorly into the midgut (Fig. 6). This surprising phenomenon, although immediately apparent to direct observation, was further confirmed by counting the number of 5-HT branches and varicosities in the midgut. The number of varicosities and branches in the midgut of $D f D d c$ larvae was $280 \pm 18$ and $46.0 \pm 3.7(n=$ 20 ), respectively, which were more than 2 -fold that of the wild type (Fig. 7). Although variability was high and there was some overlap between wild-type and mutant samples, mean values differed significantly $(p<0.001)$, and in the majority of samples, the differences were obvious. This abnormally extensive arborization was not observed in heterozygotes $(\mathrm{Ddc} / \mathrm{CyO}, \mathrm{Df} / \mathrm{CyO})$. In fact, the mean number of varicosities and branches was 141 \pm 19 and $25.4 \pm 2.4(n=9)$ in $D d c / C y O$. In $D f / C y O$, they were $142 \pm 19$ and $18.1 \pm 2.0(n=10)$. These numbers were statistically different from $D f D d c$ mutants $(p<0.001)$ but not significantly different from the wild type.

Whether the extensive arborization of 5-HT neurons was a general phenomenon associated with all 5-HT neurons was difficult to assess because of the high density of 5-HT-containing projections at sites other than the gut (neuropil in the CNS, pharyngeal muscles, frontal ganglion).

The increase in arborization seemed to be specific to 5-HT immunoreactive fibers. Figures $4, D, E, F$, and $5 B$, show views of the glutamate and FMRFamide-like immunoreactivities in the mutant at regions analogous to the ones shown for wild type (Figs. 4, $A, B, C$, and $5 A$ ). We found that both FMRFamidelike and glutamate-like immunoreactivities in the proventriculus and midgut were very similar in controls and mutant samples. Because glutamate-like immunoreactivity is so profuse in the gut and because both the glutamate and FMRFamide staining give a considerable background, such parameters as number of branches and varicosities were not measured. Therefore, the possibility that there exist small differences between the immunoreactivities of wild-type and mutant samples cannot be excluded.

\section{The mutant phenotype is partially rescued by postembryonic increase of dopamine but not of 5-HT levels}

In order to see if increasing the levels of 5-HT or dopamine in the CNS of $D f D d c$ mutants will suppress the abnormally extensive arborization in the proventriculus and midgut, newly hatched mutant larvae were reared in a medium containing $10^{-2} \mathrm{M} 5-\mathrm{HT}$ or dopamine and analyzed at the third instar stage. We found that ingested 5-HT was taken up by 5 -HT neurons in the CNS and by fibers in the normal location of 5-HT projections, i.e., pharyngeal muscles, frontal ganglion, proventriculus, and midgut. However, the intensity of the fluorescence was considerably lower than in wild type, indicating low levels of 5-HT (Fig. 8A). To quantify the extent of the arborization of 5-HT fibers in the midgut of 5-HT-fed mutant larvae, the CNS and digestive system were dissected and incubated as described in the presence of exogenous 5-HT (Fig. $8 B$ ). We found that 5-HT-fed $D f D d c$ animals were indistinguishable from $D f D d c$ animals reared in normal diet. The mean number of varicosities and branches in 5-HT-fed mutants was $302 \pm 24$ and $43.0 \pm 2.1(n=7)$, re- 

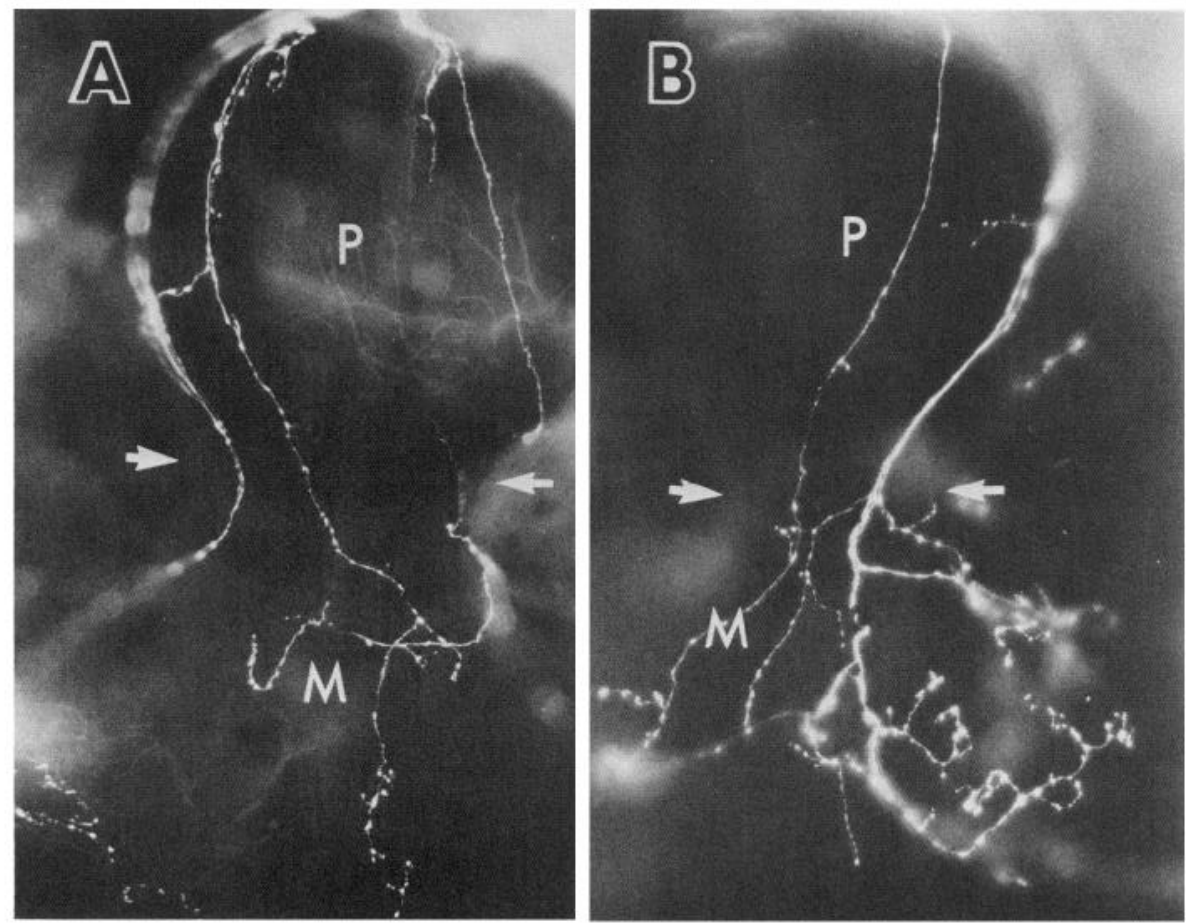

Figure 6. Distribution of 5-HT-containing fibers in the proventriculus $(P)$ and midgut $(M)$ of a wild type $(A)$ and a $D f D d c$ mutant $(B)$. This view corresponds to the area enclosed by stippled lines in Figure 1. Note the dramatic increase in the branching of the projections in the midgut of the mutant as compared to control (in these samples, the number of varicosities and branches in the midgut were 112 and 22 , respectively, for $A, 273$ and 43 for $B$ ). Some of the branches are out of focus in these photomicrographs. Arrows in $A$ and $B$ indicate the boundary between the proventriculus and midgut. $C$, Higher magnification view of 5-HT varicosities in the midgut of a wild-type larva. Scale bar, $40 \mu \mathrm{m}$ for $A$ and $B, 10$ $\mu \mathrm{m}$ for $C$.

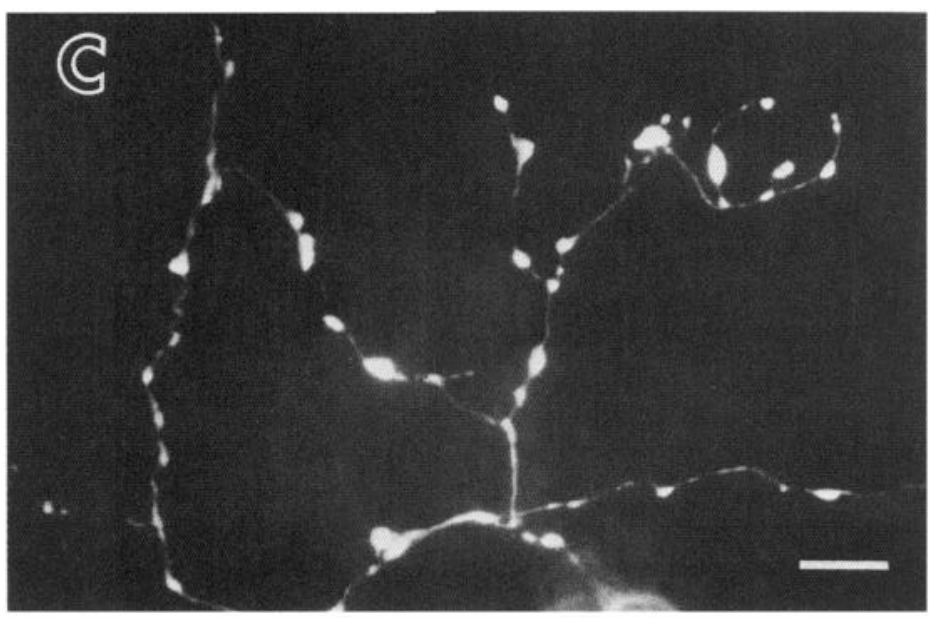

spectively, which is not significantly different from the numbers in mutants reared on normal diet (see above).

In animals reared on dopamine-containing diet, changes in the coloration of the setae and mouth parts were observed, indicating that dopamine was ingested and used in the cuticle as a precursor for tanning molecules (Fig. 9). However, since at present there is no specific anatomical probe to assess the presence of dopamine in the CNS, we do not know if dopamine was taken up by neurons in the CNS. As expected, the CNS of these animals was devoid of 5-HT immunoreactivity. Visualization of 5-HT fibers by in vitro 5-HT uptake revealed that animals reared in dopamine had a pattern of arborization in the midgut that was intermediate between the mutant and the wild type $(212 \pm 14 ; 34.0 \pm 2.3 ; n=20$, in dopamine-fed mutant animals). Statistically significant differences were found when compared both to wild type $(p<0.002)$ and to mutants reared on normal diet $(p<0.02)$. Thus, dopamine in diet, unlike 5-HT, partially rescued the mutant phenotype.
Similar to dopamine, $10^{-2} \mathrm{M} \mathrm{N}$-acetyldopamine, a metabolite in sclerotin and melanin formation (Wright, 1987), also produced changes in the pigmentation of mutant larvae. However, these animals failed to show statistically significant differences when compared with the mutant reared on normal diet, although the number of varicosities and branches in the midgut was somewhat smaller than in mutants raised on a normal diet $(237 \pm$ 27 and $39.0 \pm 5.7$, respectively; $n=9$, in N-acetyldopaminefed mutant animals; Fig. 7).

Another effect that both dopamine and $\mathrm{N}$-acetyldopamine had was to rescue the lethality of $D f D d c$ mutants at the larvalpupal boundary. As shown in Figure 10, both dopamine and $\mathrm{N}$-acetyldopamine increased the viability of $D f D d c$ larvae and allowed them to develop through eclosion. A few $D f D d c$ animals actually eclosed from the pupal case but died shortly thereafter. In addition to these effects, dopamine and $\mathrm{N}$-acetyldopamine shortened the abnormally long larval development of the mutants to wild-type values (Fig. 10, arrows). 

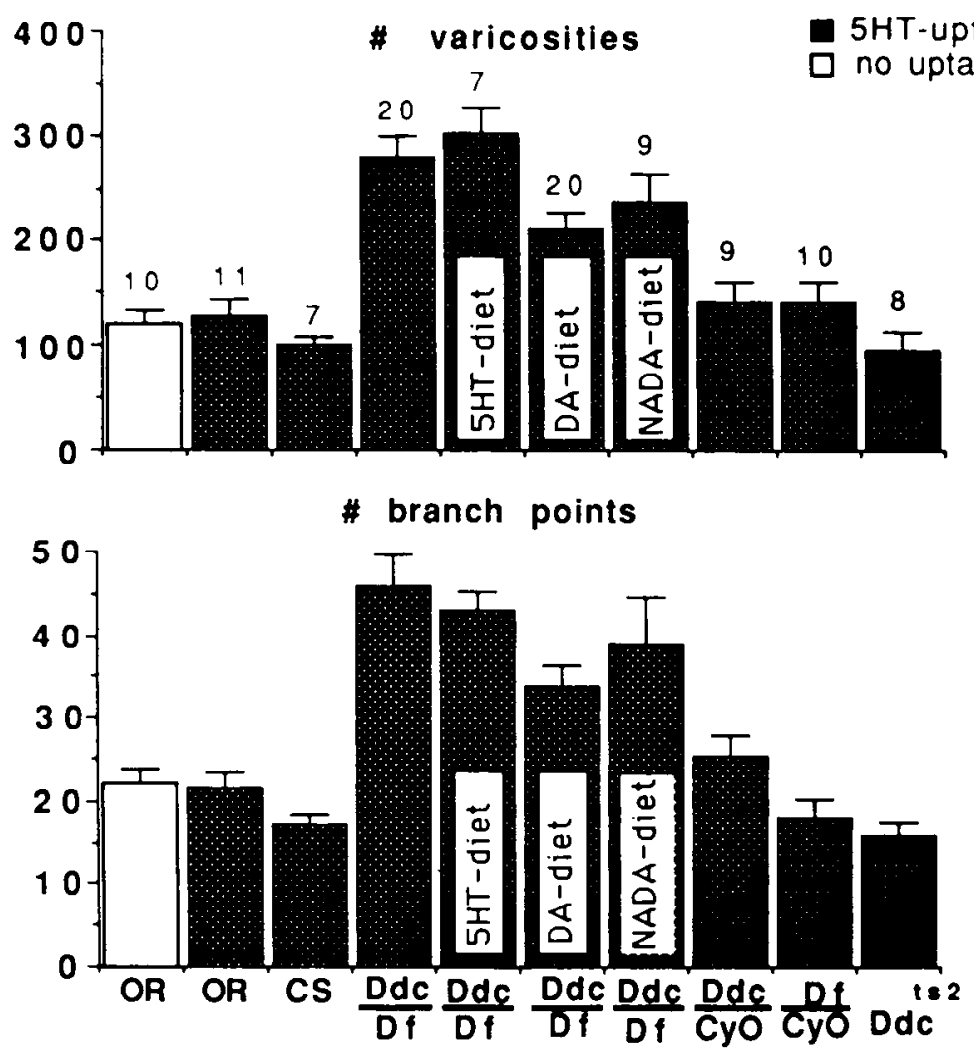

\begin{abstract}
Figure 7. Number of 5-HT branches and varicosities in the midgut of Drosophila larvae of different genotypes and subjected to different procedures. The number of samples analyzed is indicated above the SEM bars at the top histogram. The samples for the bottom histogram are the same as for the top histogram.
\end{abstract}

\section{Low levels of DDC activity are sufficient to give wild-type phenotype}

To determine if the mutant phenotype is produced in the presence of low levels of 5-HT or dopamine throughout embryonic and larval development, "leaky" $D d c$ mutants were investigated. $D d c^{t s 2}$ mutants have been reported to contain less than $3 \%$ of normal DDC enzyme activity when reared at restrictive temperature $\left(28^{\circ} \mathrm{C}\right)$ (Wright et al., 1981). However, Vallés and White (1986) have shown that 5-HT immunoreactivity can be detected in the CNS of these mutants, even when they are reared at restrictive temperature.

$D d c^{\prime s 2}$ broods were incubated at $30^{\circ} \mathrm{C}$ from 1 day before egg laying, and the eggs were collected and reared at the same temperature until dissection at the third larval stage. 5-HT immunoreactivity in these mutants is considerably lower than in wild type (not shown). However, uptake experiments reveal that the pattern of branching and the number of varicosities in the midgut of these animals is not significantly different from wild type $\left(96 \pm 17\right.$ and $15.9 \pm 1.5$, respectivcly; $n=8$; in $D d c^{s / 2}$; Fig. 7). This indicates a suppression of the mutant phenotype by low levels of DDC during embryogenesis.

\section{Discussion}

\section{Methodology}

To visualize 5-HT projections in $D f D d c$ mutants, mutant CNSs were incubated in exogenous serotonin. The immunoreactive fibers observed in the proventriculus and midgut most likely correspond to the 5-HT fibers in wild-type larvae, based on the following considerations. First, the pattern of distribution of 5-HT neurons in $D f D d c$ larvae after incubation in exogenous 5-HT is analogous to the wild-type pattern (Vallés and White, 1986). This is in agreement with what is found in other systems where the specificity of monoamine uptake systems has been studied (Axelrod, 1965; Osborne et al., 1975; Ross, 1982; Glover, 1987). Second, similar experiments performed in wild-type larval CNS revealed that neurons other than the normal 5-HT neurons do not take up 5-HT at the concentration used (Vallés and White, 1986; also in this report). However, in those experiments, attention was paid to the cell bodies, and fibers were not studied in any detail. There exists a possibility that mutant fibers other than those corresponding to the normal 5-HT fibers could take up 5-HT. That possibility is unlikely, however, because (1) fibers that take up 5-HT in the mutant are confined to the sites normally seen in wild type, and (2) the number of branches and varicosities in the midgut of wild-type larvae remains unchanged after incubation with exogenous $5-\mathrm{HT}$.

\section{Aberrant 5-HT fibers in DfDdc}

An interesting result of this study is that although $D f D d c$ mutants are devoid of 5-HT, 5-HT fibers and presumably synaptic contact regions do form in the appropriate targets, as indicated by the large number of varicosities. In a number of vertebrate and invertebrate systems, varicosities have been suggested as regions of presynaptic contacts (Muller and MacMahan, 1976; DeReimer and Macagno, 1981; Murphey et al., 1983; Murphey and Lemere, 1984). Further physiological and ultramicroscopic analysis would be necessary to assess the functionality of this innervation in the mutant. A possibility is that these fibers may contain another neurotransmitter/neuromodulator in addition to 5-HT. Colocalization of transmitters has been reported in an increasing number of preparations (Lloyd et al., 1981; Lundberg, 1981; Osborne et al., 1982; Adams and O'Shea, 1983; Lundberg and Hökfelt, 1983; Cournil et al., 1984; Siwicki et al., 1987; Hökfelt et al., 1987). In this study, we found that glutamate and FMRFamide-like peptide(s) are also present in the proven- 

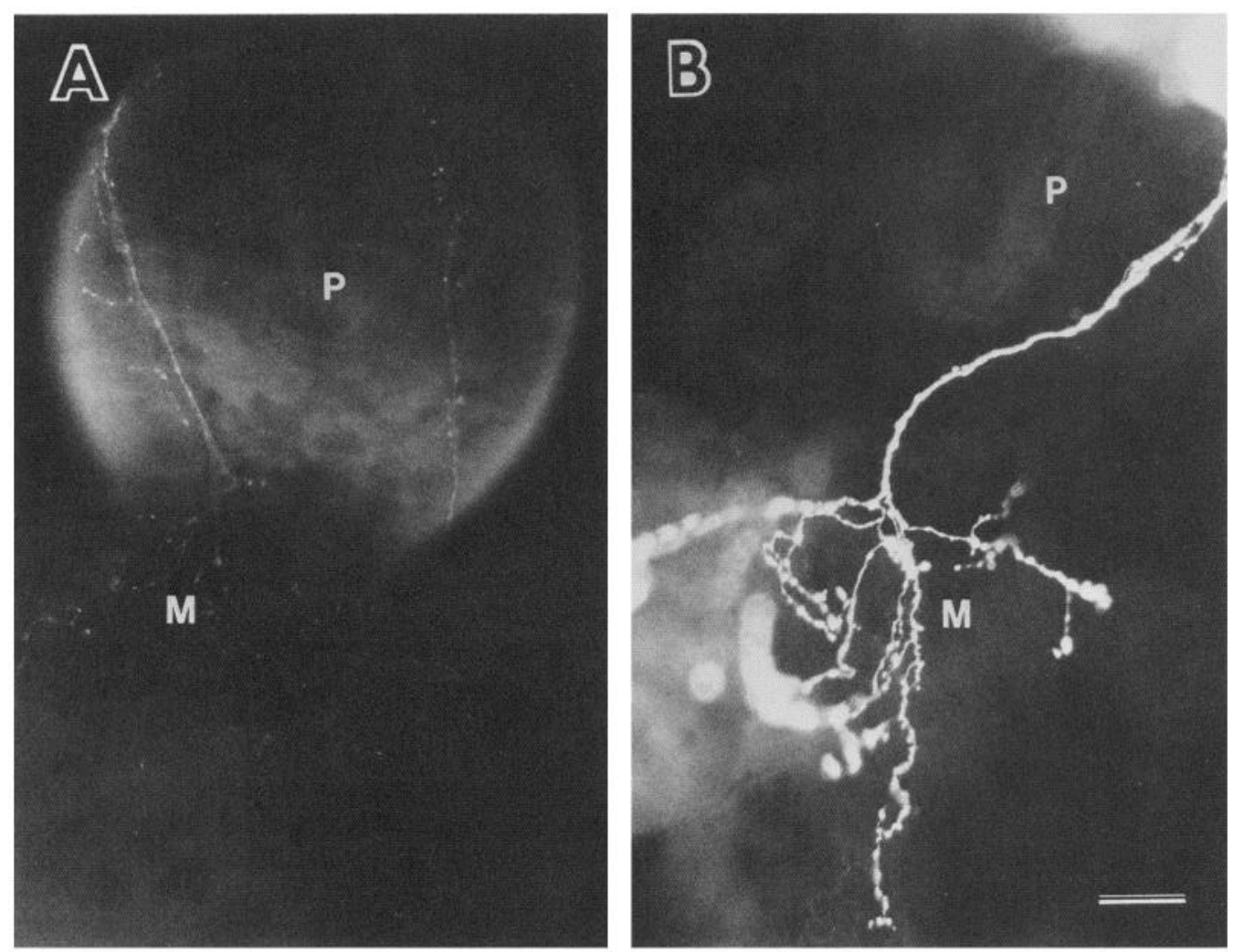

Figure 8. 5-HT immunoreactivity in $D f D d c$ larval gut $(A)$, of a 5-HT-fed third instar larva (see text), and $(B)$ of a 5-HT-fed third instar larva incubated in exogenous 5-HT after dissection. Note the extensive arborization of fibers comparable to the one observed in the gut of $D f D d c$ animals reared in normal diet (Fig. 6; in this sample, the number of varicosities and branches was 314 and 37, respectively). $P$, proventriculus; $M$, midgut. Scale bar, $40 \mu \mathrm{m}$.

triculus and midgut. However, they appear to correspond to different, nonoverlapping subsets.

An unexpected feature of the 5-HT fibers in the proventriculus and midgut of $D f D d c$ mutants is their dramatically increased arborization. Counts of branches and varicosities in the midgut reveal approximately a 2 -fold difference in the mean. This phenomenon is not seen in heterozygotes, nor in 2 wild-type strains of Drosophila. To produce $D f D d c$ animals, we used 2 independently generated parental strains carrying mutations for the gene $D d c\left[D d c^{n 27} / C y O\right.$, and $\left.D f(2 L) 130 / C y O\right]$. Therefore, the increased arborization in the mutants is not likely to be due to a recessive mutation other than $D d c$ at another location in the genome.

Our studies are in agreement with what Kater et al. have found in cultured neurons of Helisoma. In their in vivo studies, they showed that lowering levels of 5-HT at a given developmental stage produces an abnormal, extensive arborization in a specific, identified neuron (Goldberg and Kater, 1989). They proposed that 5-HT and dopamine could serve as factors that regulate elongation of neurites. Analogous results have been obtained in raphe neurons in the rat and in a population of chick retinal neurons (Davila-Garcia et al., 1985; Lankford et al., 1988).

The increased extent of arborization of fibers found in the midgut of $D f D d c$ seems to be specific to 5-HT fibers. We found no apparent difference in the distribution of glutamate and FMRFamide immunoreactivity in the proventriculus and midgut in mutant and control animals. We were not able to determine if the alteration is in all 5-HT fibers of the mutant nervous system (pharyngeal muscles, frontal ganglion, neuropil in the CNS). The high density of fibers and varicosities at these sites or the presence of skeletal elements (cephalopharyngeal plates over the pharyngeal muscles) prevented a quantitative analysis.

\section{Monamine feeding experiments}

Since $D f D d c$ mutants are devoid of 5-HT and dopamine, we attempted to manipulate the levels of these molecules by feeding mechanically hatched larvae with monoamines and by using a temperature-sensitive allele of the $D d c$ gene $\left(D d c^{t s 2}\right)$. We found that feeding 5-HT to mutant larvae indeed resulted in the incorporation of this amine into the CNS, as revealed by immunocytochemical staining. The resultant fluorescence was lower than in wild type but above the threshold of detection, especially in the fibers of the pharyngeal muscles, frontal ganglion, proventriculus, and midgut. 5-HT uptake experiments performed in these 5-HT-fed mutants revealed that an increase in the levels of 5-HT at larval stages did not rescue the mutant phenotype. Since 5-HT fibers at the proventriculus and midgut start developing before hatching, it would be interesting to see if increasing the levels of 5-HT during the embryonic period would have any effect.

In contrast to 5-HT feeding experiments, dopamine feeding did partially rescue the mutant phenotype. Quantitative analysis revealed that the number of fibers and varicosities in the proventriculus and midgut is between the wild type and the mutant values and is statistically different from both of them. In contrast, feeding with a $\mathrm{N}$-acetyldopamine-containing diet did not 
significantly change the mutant phenotype in the proventriculus and midgut. Both dopamine and $\mathrm{N}$-acetyldopamine, however, changed the pigmentation and increased the viability of the larvae. This could imply that the change in the extent of branching seen in the mutant is caused by the absence of dopamine itself and not by a metabolite located further along the catecholamine metabolic pathway. However, this result should be analyzed with caution, since the statistical test failed in finding a difference between the numbers obtained from dopamine-fed and $\mathrm{N}$-acetyldopamine-fed mutants. Since other catecholamine metabolites were not used, further experiments will be necessary to test this point.

To further examine the roles of dopamine and 5-HT, it would be interesting to determine if ple mutants also show this phenotype (Budnik and White, 1987a). These mutants have CNSs devoid of catecholamines, but the levels of 5-HT seem to be unaltered. Unfortunately, these mutant animals die by late embryogenesis, and we found that comparison of fibers at this stage is technically very difficult.

Several possibilities may explain the action of dopamine in the proventriculus and midgut of $D f D d c$.

1. As suggested by McCobb et al. (1988), dopamine could serve as one of the factors regulating the cessation of neurite elongation on some specific neurons. In the absence of dopamine, 5-HT fibers continue growing and ramifying. To demonstrate this hypothesis, several conditions should be met. First, a source of dopamine should be normally present during development near the 5-HT fibers. Studies of catecholamine-containing neurons in Drosophila larvae reveal that there are no peripheral catecholamine-containing projections (Budnik et al., 1986; Budnik and White, 1988). However, dopamine could be released from the CNS to the hemolymph by a neurohormonal system. Neurohemal organs that release hormones to the hemolymph have been found in a number of insect preparations (Flanagan, 1984; Davis, 1985). The second condition is that fibers in the proventriculus and midgut or the target that they innervate should have receptors for dopamine. Third, the release of dopamine to these sites should overlap with the growth period of the neurites. Another alternative is that the neurites themselves could have a defined period of sensitivity to dopamine.

An interesting observation is that 5-HT fibers in the proventriculus and midgut start developing before hatching in both wild-type and $D f D d c$ mutants. The fact that increase in postembryonic levels of dopamine partially rescued the mutant phenotype implies that modulation of neurite outgrowth can still be attained after the fibers reach their targets. Dopamine could cause retraction of previously formed ramifications or arrest of neurite outgrowth at early larval stages. This latter possibility would account for the partial rather than full rescue of the mutant phenotype. Since it is difficult to determine whether the mutant phenotype was already present by hatching, these two possibilities cannot be discerned.

2. Another hypothesis that could explain the anatomical abnormality found in the $D f D d c$ mutant is that this effect is an epiphenomenon due to the lack of dopamine in the CNS. The mutant phenotype, for example, could be caused by the lack of the physiological effect that dopamine normally has in the target, or, alternatively, the lack of the neurotransmitter could produce changes at the level of the whole animal. However, weakening this hypothesis is the fact that the defect is specific to 5-HT fibers and not found in glutamate and FMRFamide systems.
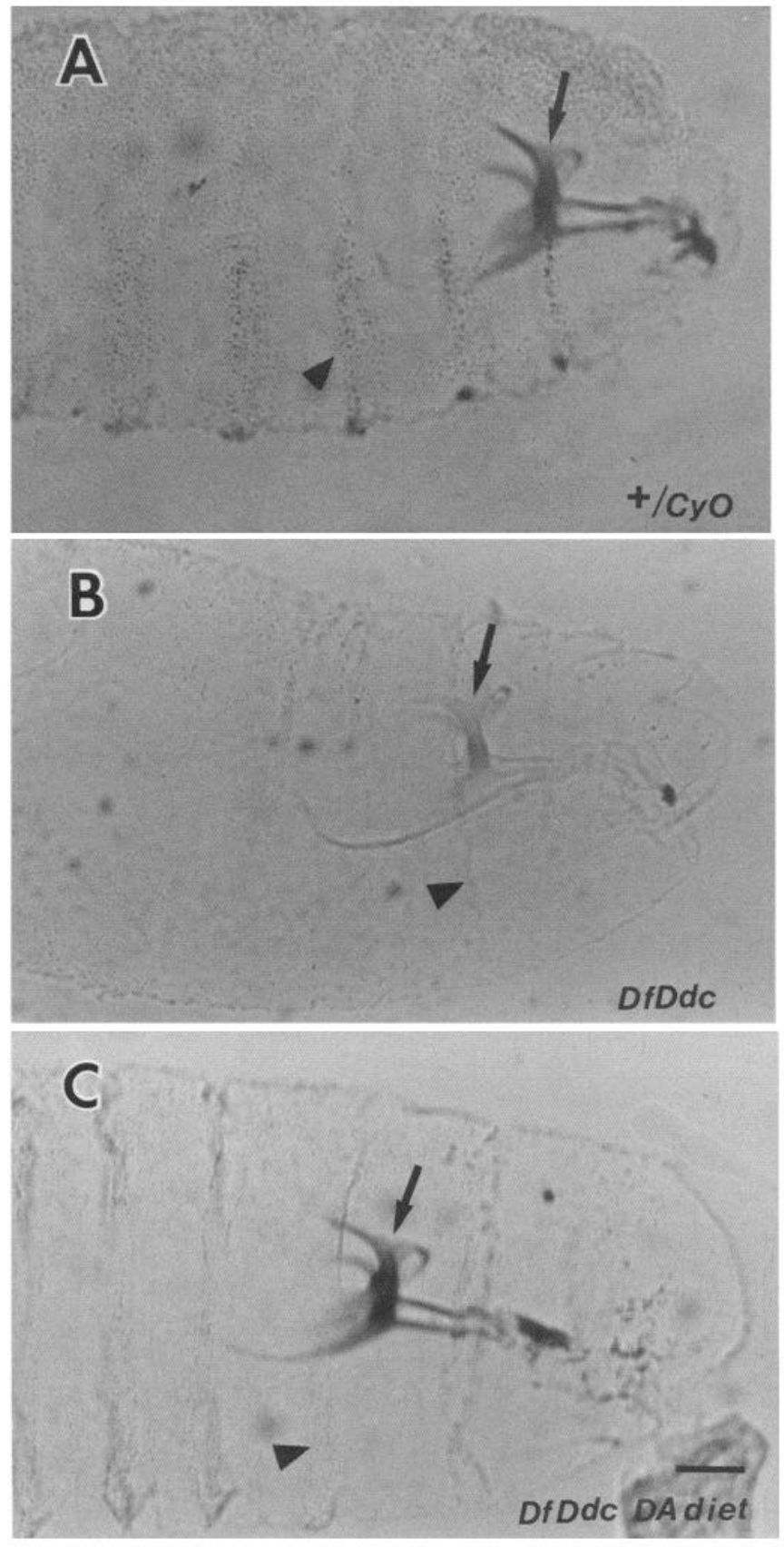

Figure 9. Cephalopharyngeal plates (arrow) and setae (arrowhead) of a wild-type second instar larva $(A)$, a $D f D d c$ larva $(B)$, and a dopaminefed $D f D d c$ larva $(C)$. Notice the absence of pigmentation in the cephalopharyngeal plates and setae in $B$ and the reappearance of some of this pigmentation in $C$. Scale bar, $50 \mu \mathrm{m}$.

Furthermore, $\mathrm{N}$-acetyldopamine diet results in increased viability, yet the mutant phenotype in the gut is not rescued.

In conclusion, we have found that in the absence of dopamine and 5-HT, identified 5-HT fibers develop and project to their appropriate target area. These fibers, however, show an aberrant pattern of arborization. This abnormality can be partially rescued by feeding $D f D d c$ pharate larvae with dopamine, but not with 5-HT. 
Figure 10. Percentage of surviving wild-type and mutant animals after mechanical hatching, subjected to different treatments. Wandering stage $(w)$ and eclosion $(e)$ are indicated by the arrows. The number of mechanically hatched animals for each experimental group is: $D f D d c=132 ; D f D d c 5-\mathrm{HT}$ diet $=86$; $D f D d c$ dopamine diet $=118 ; D f D d c$ $\mathrm{N}$-acetyldopamine diet $=105 ; \mathrm{Ddc} / \mathrm{CyO}$ $=48$.

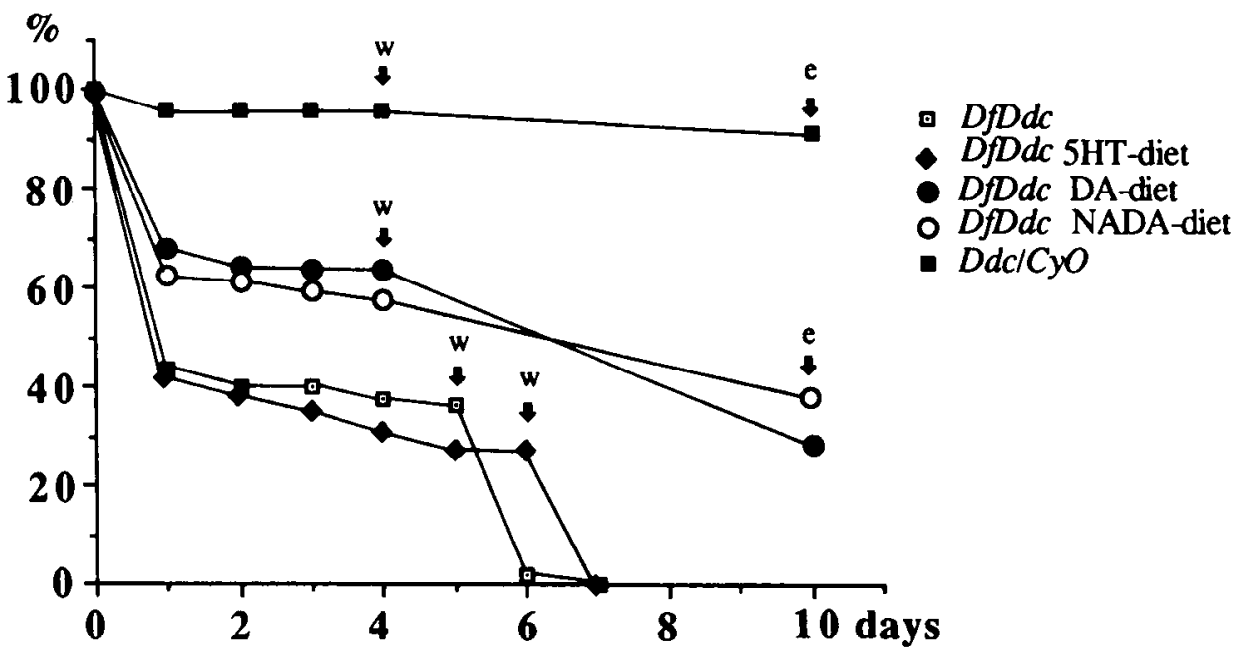

\section{References}

Adams, M. E., and M. O'Shea (1983) Peptide cotransmitter at a neuromuscular junction. Science 221: 286-290.

Axelrod, J. (1965) The metabolism, storage and release of catecholamine. Recent Prog. Horm. Res. 21: 597-622.

Rear, M. F., and W. Singer (1986) Modulation of visual cortical plasticity by acetylcholine and noradrenaline. Nature 320: 172-176.

Bodenstein, D. (1950) The post-embryonic development of Drosophila. In Biology of Drosophila, M. Demerec, ed., pp. 275-367, Hafner, New York.

Budnik, V., and K. White (1987a) Genetic dissection of dopamine and serotonin synthesis in the nervous system of Drosophila melanogaster. J. Neurogenet. 4: 309-314.

Budnik, V., and K. White (1987b) A serotonergic system in Drosophila melanogaster. Soc. Neurosci. Abstr. 13: 234.

Budnik, V., and K. White (1988) Catecholamine-containing neurons in Drosophila melanogaster: Distribution and development. J. Comp. Neurol. 268: 400-413.

Budnik, V., L. Martin-Morris, and K. White (1986) Perturbed pattern of catecholamine-containing neurons in mutant Drosophila deficient in the enzyme dopa decarboxylase. J. Neurosci. 6: 3682-3691.

Budnik, V., C.-F. Wu, and K. White (1988) Altered branching of serotonin-containing neurons in Drosophila mutants unable to synthesize serotonin and dopamine. Soc. Neurosci. Abstr. 14: 415.

Campos-Ortega, J. A., and V. Hartenstein (1985) The Embryonic Development of Drosophila melanogaster, Springer-Verlag, New York.

Cournil, I., M. Geffard, M. Moulins, and M. LeMoat (1984) Coexistence of dopamine and serotonin in an identified neuron of the lobster nervous system. Brain Res. 310: 397-400.

Davila-Garcia, M. I., P. Alvarez, P. M. Whitaker-Azmitia, A. C. Towle, and E. C. Azmitia (1985) Effects of serotonin and 5-methoxytryptamine on GABA and 5-HT immunostained cells from the hippocampus and mesencephalic raphe grown in tissue culture. Soc. Neurosci. Abstr. 11: 601 .

Davis, N. T. (1985) Serotonin-immunoreactive visceral nerves and neurohemal system in the cockroach Periplaneta americana. Cell Tissue Res. 240: 593-600.

DeReimer, S. A., and E. R. Macagno (1981) Light microscopic analysis of contacts between pairs of identified leech neurons with combined use of horseradish peroxidase and lucifer yellow. J. Neurosci. 1: 650657.

Flanagan, T. R. J. (1984) Cytological evidence for serotonin-containing fibers in an abdominal neurohemal organ in an hemipteran. Brain Res. 306: 235-242.

Gilbert, D. J., J. Hirsh, and T. R. F. Wright (1984) An analysis of dopa decarboxylase expression during embryogenesis in Drosophila melanogaster. Dev. Biol. 107: 142-155.

Glover, J.C. (1987) Serotonin storage and uptake by identified neurons in the leech Hementeria ghilianii. J. Comp. Neurol. 256: 117-127.

Goldberg, J. I., and S. B. Kater (1989) Expression and function of the neurotransmitter serotonin during development of the Helisoma nervous system. Dev. Biol. 131: 483-495.

Gorczyca, M. G., and J. C. Hall (1987) Immunocytochemical localization of choline acetyltransferase during development and in $C h a^{\text {ss }}$ mutants of Drosophila melanogaster. J. Neurosci. 7: 1361-1369.

Greenspan, R. J. (1980) Mutations of choline acetyltransferase and associated neural defects in Drosophila melanogaster. J. Comp. Physiol. 137: 83-92.

Greenspan, R. J., J. A. Finn, and J. C. Hall (1980) Acetylcholinesterase mutants in Drosophila and their effects on the structure and function of the central nervous system. J. Comp. Neurol. 189: 741-774.

Harris, W. A. (1982) Neural activity and development. Annu. Rev. Physiol. 43: 689-710.

Haydon, P. G., D. P. McCobb, and S. B. Kater (1984) Serotonin selectively inhibits growth cone motility and synaptogenesis of specific identified neurons. Science 226: 561-564.

Haydon, P. G., D. P. McCobb, and S. B. Kater (1987) The regulation of neurite outgrowth, growth cone motility, and electrical synaptogenesis by serotonin. J. Neurobiol. 18: 197-215.

Hepler, J. R., C. S. Toomim, K. D. McCarthy, F. Conti, G. Battaglia, A. Rustioni, and P. Petrusz (1988) Characterization of antisera to glutamate and aspartate. J. Histochem. Cytochem. 36: 13-22.

Hirsh, J. (1986) The Drosophila melanogaster dopa decarboxylase gene: Progress in understanding the in vivo regulation of a higher eukariotic gene. In Molecular Developmental Biology, L. Bogorad, ed., pp. 103-114, A. R. Liss, New York.

Hökfelt, T., D. Millhorn, K. Seroogy, Y. Tsuruo, S. Ceccatelli, B. Lindh, B. Meister, T. Melander, M. Schalling, T. Bartfai, and L. Terenius (1987) Coexistence of peptides with classical neurotransmitters. Experientia 43: 768-780.

Johansen, J., M. E. Halpern, K. M. Johansen, and H. Keshishian (1989) Stereotypic morphology of glutamatergic synapses on identified muscle cells of Drosophila larvae. J. Neurosci. 9: 710-725.

Johnson, W. A., C. J. Beall, S. J. Bray, B. A. Morgan, C. A. McCormick, and J. Hirsh (1987) Neuronal and glial expression of the Drosophila melanogaster dopa decarboxylase gene. Soc. Neurosci. Abstr. 13:559.

Lankford, K. L., F. G. Demello, and W. L. Klein (1988) D1-type dopamine receptor inhibits growth cone motility in cultured retina neurons: Evidence that neurotransmitters act as morphogenic growth regulators in the developing central nervous system. Proc. Natl. Acad. Sci. USA 85: 2839-2843.

Lauder, J. M., and H. Krebs (1978) Serotonin as a differentiation signal in early neurogenesis. Dev. Neurosci. 1: 15-30.

Lauder, J. M., and H. Krebs (1984) Humoral influences on brain development. Adv. Cell Neurobiol. 5: 3-51.

Lindsley, D. L., and E. H. Grell (1968) Genetic Variations of Drosophila melanogaster. Carnegie Institution of Washington Publication No. 627.

Livingstone M.S., and B. L. Tempel (1983) Genetic dissection of monoamine neurotransmitter synthesis in Drosophila. Nature 303: 67-70. 
Lloyd, P. E., B. P. Masinovsky, R. E. McCaman, and A. O. D. Willows (1981) Coexistence of neuropeptide and acetylcholine in identified molluscan neuron. Soc. Neurosci. Abstr. 7: 637.

Lundberg, J. M. (1981) Evidence for coexistence of vasoactive intestinal polypeptide (VIP) and acetylcholine in neurons of cat exocrine glands. Acta Physiol. Scand. 112 (suppl. 496): 1-57.

Lundberg, J. M., and T. Hökfelt (1983) Coexistence of peptides and classical neurotransmitters. Trends Neurosci. 6: 325-333.

Marder, E., R. L. Calabrese, M. P. Nusbaum, and B. Trimmer (1987) Distribution and partial characterization of FMRFamide-like peptides in the stomatogastric nervous systems of the rock crab, Cancer borealis, and the spiny lobster, Panulirus interruptus. J. Comp. Neurol. 259: 150-163.

McCobb, D. P., P. G. Haydon, and S. B. Kater (1988) Dopamine and serotonin inhibition of neurite elongation of different identified neurons. J. Neurosci. Res. 19: 19-26.

Mercier, A. J., P. G. Haydon, and S. B. Kater (1985) A role for serotonergic neurons in the regulation of specific electrical synapses in Helisoma. Soc. Neurosci. Abstr. 11: 614.

Muller, K. J., and U. J. MacMahan (1976) The shapes of sensory and motor neurons and the distribution of their synapses in the ganglia of the leech: A study using intracellular injection of horseradish peroxidase. Proc. R. Soc. Lond. (Biol.) 194: 481-499.

Murphey, R. K, and C. A. Lemere (1984) Competition controls the growth of an identified axonal arborization. Science 228: 1352-1355.

Murphey, R. K., S. E. Johnson, and D. S. Sakaguchi (1983) Anatomy and physiology of supernumerary cercal afferents in crickets: Implications for pattern formation. J. Neurosci. 3: 312-325.

Osborne, N. N., L. Hiripi, and V. Neuhoff (1975) The in vitro uptake of biogenic amines by snail (Helix pomalia) nervous system. Biochem. Pharmacol. 24: 2141-2148.

Osborne, N. N., A. C. Cuello, and G. J. Dockray (1982) Substance P and cholecystokinin-like peptides in Helix neurons and cholecystokinin and serotonin in a giant neuron. Science 216: 409-411.
Pettigrew, D. J., and T. Kasamatsu (1976) Local perfusion of noradrenaline maintains visual cortical plasticity. Nature 271: 761-763.

Russ, S. B. (1982) The characteristics of serotonin uptake systems. In Biology of Serotonergic Transmission, N. N. Osborne, ed., pp. 159196, Wiley, New York.

Siwicki, K. K., S. B. Beltz, and E. Kravitz (1987) Proctolin in identified serotonergic, dopaminergic, and cholinergic neurons in the lobster, Homarus americanus. J. Neurosci. 7: 522-532.

Vallés, A. M., and K. White (1986) Development of serotonin-containing neurons in Drosophila mutants unable to synthesize serotonin. J. Neurosci. 6: 1482-1491.

Vallés, A. M., and K. White (1988) Serotonin-containing neurons in Drosophila melanogaster: Distribution and development. J. Comp. Neurol. 268: 414-428.

Van der Meer, J. (1977) Optical clean and permanent whole mount preparation for phase contrast microscopy of cuticle structures of insect larvae. Drosophila Inf. Serv. 52: 160.

White, K., T. Hurteau, and P. Punsal (1986) Neuropeptide-FMRFamide-like immunoreactivity in Drosophila: Development and distribution. J. Comp. Neurol. 247: 430-438.

Wright, T. R. F. (1987) The genetics of biogenic amine metabolism, sclerotization and melanization in Drosophila melanogaster. Adv. Genet. 24: 127-222.

Wright, T. R. F., R. B. Hodgetts, and A. F. Sherald (1976) The genetics of dopa decarboxylase in Drosophila melanogaster. I. Isolation and characterization of deficiencies that delete the dopa decarboxylase dosage-sensitive region and the alpha-methyl-dopa hypersensitive locus. Genetics 84: 267-285.

Wright, T. R. F., R. Steward, K. W. Bentley, and P. A. Adler (1981) The genetics of dopa decarboxylase in Drosophila melanogaster. III. Effects of a temperature-sensitive dopa decarboxylase-deficient $\mathrm{mu}$ tation on female fertility. Dev. Genet. 2: 223-235. 